\title{
EXPLORING GEOSPATIAL VARIATION IN DIABETES-RELATED PRIMARY HEALTH CARE SERVICE UTILISATION AND POTENTIALLY PREVENTABLE HOSPITALISATIONS IN WESTERN AUSTRALIA
}

\author{
Bert Veenendaal ${ }^{1 *}$, Colleen Koh ${ }^{2}$, Ashty Saleem ${ }^{14}$, Richard Varhol ${ }^{34}$, Jianguo Xiao ${ }^{2}$, Bella Mai ${ }^{2}$, Yudan Liu ${ }^{2}$ \\ ${ }^{1}$ Spatial Sciences, Curtin University, GPO Box U1987, Perth, Australia - (b.veenendaal, a.saleem)@curtin.edu.au \\ ${ }^{2}$ Department of Health Western Australia, 189 Royal Street, East Perth, Australia - (Jianguo.Xiao, Colleen.Koh, Qun.Mai, \\ Yuna.Liu)@health.wa.gov.au \\ ${ }^{3}$ School of Public Health, Curtin University, GPO Box U1987, Perth, Australia - rvarhol@curtin.edu.au \\ ${ }^{4}$ Cooperative Research Centre for Spatial Information, Australia
}

\author{
Commission IV, WG IV/3
}

KEY WORDS: Health, diabetes, potentially preventable hospitalisation, geospatial analysis, spatial analysis, GIS

\begin{abstract}
:
Greater investments and improvements in primary health care (PHC) can provide benefits in reducing the high costs of hospital admissions. Potentially preventable hospitalisations (PPH) are a health system performance indicator used to evaluate access to and effectiveness of community-based health services. The Western Australia Department of Health obtained detailed primary health care data, for the first time at the postcode level scale, and analysed its associations with PPH information for selected conditions. PHC data obtained from the Commonwealth Department of Health for the financial year 2013/14 was Medicare Benefits Schedule (MBS) and Pharmaceutical Benefits Scheme (PBS) records at postcode level. In this paper we explore the sensitivity of various benchmarks of spatial zonings for comparison of diabetes-related primary health care utilisation and potentially preventable hospitalisations and then examine the relationship between them among the various spatial zonings. From the geospatial visualisation and analysis undertaken, conclusions are drawn about the patterns and relationships between diabetes-related primary health care utilisation and potentially preventable hospitalisations. The scale of spatial zonings used for comparison is important as too large or too small areas may mask out the relative geospatial variation of diabetes-related PHC utilisation and PPH evident among postcode areas.
\end{abstract}

\section{INTRODUCTION}

\subsection{Background}

Greater investments and improvements in primary health care (PHC) can provide benefits in reducing the high costs of hospital admissions (DOHWA 2017). Potentially preventable hospitalisations $(\mathrm{PPH})$ are a health system performance indicator used to evaluate access to and effectiveness of community-based health services (Falster and Jorm 2017). PPH identifies cases of hospital admissions where "the hospitalisation could potentially have been prevented through the provision of appropriate individualised preventative health interventions and early disease management, usually delivered in primary care and community-based care settings" (p. 3, Falster \& Jorm 2017). However, being able to measure the benefits of improved PHC and subsequent reduction in PPHs is a challenge.

The Western Australia Department of Health Western Australia obtained detailed primary health care data, for the first time at the postcode level scale, and analysed its associations with PPH information for selected conditions. Specifically, the PHC data obtained was Medicare Benefits Schedule (MBS) and Pharmaceutical Benefits Scheme (PBS) records. The MBS data captures Medicare benefits claims for clinically relevant services provided by an appropriate health practitioner (AGDOH 2011). The PBS data captures the prescription records written by an authorised PBS prescriber for a medicinal product included in the schedule of the PBS which is dispensed by an approved supplier (AGDOH 2018). Data pertaining to the MBS and PBS were obtained from the Commonwealth Department of Health for the financial year 2013/14.

Some initial descriptive and statistical analysis including an investigation of geospatial patterns of variation of MBS and PBS usage in relation to PPHs for a range of health conditions was explored by Gavidia et al. (2018). This work was extended for this paper by focussing on the geospatial variation in patterns specifically for diabetes across Western Australia.

\subsection{Overview}

This paper focuses on the sensitivity of spatial zoning for comparison of diabetes-related PHC utilisation and PPH. Spatial unit boundaries are simply proxies for individuals residing within those boundaries and may be arbitrary and not accurately reflecting the individuals (Duckett and Griffiths 2016). Smaller units may more accurately reflect the underlying population distribution. We use postcode areas to represent PHC and PPH, and compare with larger spatial units to better understand geospatial (or simply "spatial") variation.

Previous work by Gavidia et al. (2018) compared diabetesrelated (and other medical conditions) PHC utilisation with PPHs for postcode areas relative to State (of Western Australia) rates. Analysis of the data suggests that lower PHC utilisation together with higher PPHs occurs predominantly in rural

\footnotetext{
* Corresponding author
} 
regions whereas lower PHC utilisation and lower PPHs tends to occur mainly in Perth metropolitan and country regions that are least socioeconomically disadvantaged or have low general practice ratios. Higher PHC utilisation and higher PPHs appear in more socioeconomically disadvantaged metropolitan and rural areas.

To better understand the spatial variation, we explored the comparison of PHC utilisation and PPHs for postcode areas relative to smaller, more localised regions, in addition to comparing to the state as a whole. In particular we first explored three regions: metropolitan, regional and other rural, as a basis for comparison, given that each of these three regions has different levels of access to health services. We then modified these regions using the Accessibility/Remoteness Index of Australia (ARIA+) national standard classification (DOHAC 2001). We subsequently explored the use of the administrative Health Regions (HR) that are defined and utilised by the Department of Health Western Australia. Utilising geospatial approaches, these health regions were mapped and compared with each other to understand how sensitive the different scales of spatial zonal units were with regards to the pattern of PHC utilisation and PPHs. We then explored the relationship between $\mathrm{PHC}$ and $\mathrm{PPH}$ using geospatial approaches.

This paper also outlines our methodologies used for exploring sensitivity to different spatial zonings and a comparison and analysis of the results, using a range of spatial analytics tools such as hotspot and autocorrelation analyses, for identifying, exploring and visualising spatial patterns. This investigation draws out the geographic variations and relationships between PHC and PPH across the state of Western Australia and within the metropolitan region of Perth. Conducting and understanding such spatial analyses are important for health service planning, health program development and in improving PHC through the reduction of PPHs.

\section{METHODOLOGY}

\subsection{Overview of methods}

The overall methodology used for this research is outlined in Figure 1. Firstly, the PHC and PPH patient numbers for each postcode area were mapped relative to a benchmark region.

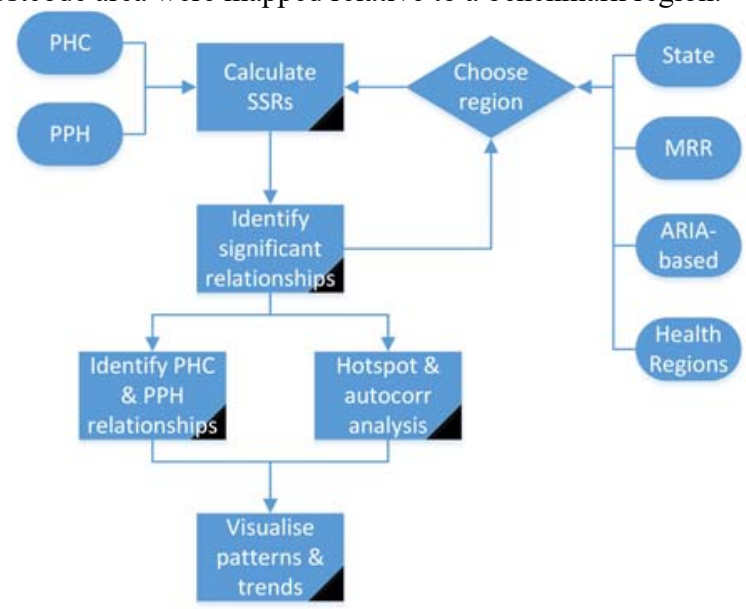

Figure 1. Methodology for exploring geospatial variation in diabetes health data

These patterns were then explored and further analysed using spatial hotspot and autocorrelation analysis. The relationships between PHC and PPH were then identified and mapped so that patterns and trends could be visualised and examined.

As PHC and PPH data each had a unique patient identification but had no common patient identification within each dataset, each patient was counted only once for the whole study period; this is referred to as a prevalent case. For each of the 381 postcodes, standardised rate ratios (SRRs) were calculated for PHCs and PPHs, respectively and obtained for a range of different benchmark regions.

The SRR is the ratio of observed PHC or PPH prevalent cases in the postcode, to expected $\mathrm{PHC}$ or PPH prevalent cases based on the selected state/regional rate. The SRRs were calculated using the indirect standardisation method (Rothman and Greenland, 1998). The statistical significance of the SRRs was mapped indicating whether the PHC or PPH occurrence was significantly higher than, lower than or similar to the benchmark region averages. The resulting patterns were then visualised and compared for the different regions used.

The spatial patterns of PHC and PPH were further explored by performing a hotspot analysis and a Local Moran's I autocorrelation analysis on the significance values, and then mapping the results to examine the patterns. Hotspot analysis identifies hot and cold spots, which are, in this case, postcodes of high and low, respectively, incidence in comparison to surrounding postcodes (Duckett and Griffiths 2016). Local autocorrelation analysis shows how the PHC and PPH SRR values for a postcode relate to neighbouring postcodes around it (Duckett and Griffiths 2016). Postcodes may be clustered within high (High-High) or low neighbourhoods (Low-Low), or be outliers among high (Low-High) or low (High-Low) neighbourhoods.

What is more important than exploring the spatial patterns of PHC and PPH separately, is exploring them together. We were interested in 5 relationships based on the potential implications for health services provision; these include four distinct relationships used in the work done by Gavidia et al. (2018), together with the case where PPH and PHC are similar. These are as follows:

\section{1 - lower PPH, lower PHC \\ 2 - lower PPH, higher PHC \\ 3 - similar PPH, similar PHC \\ 4 - higher PPH, lower PHC \\ 5 - higher $\mathrm{PPH}$, higher $\mathrm{PHC}$}

These five classes were used as a possible ranking to indicate lower values (eg. 1 and 2) as more preferred relationships and higher values (eg. 4 and 5) as being less preferred. These rankings are debatable as a lower $\mathrm{PHC}$ can mean not just lower usage of PHC services, but a lack of access to PHC services, and so it could be argued that lower PPH and higher PHC should be highest priority. Although not part of this research, the rankings can be altered for future further analysis. By ranking the classes, we were able to undertake some further spatial analysis.

This is then mapped and the underlying pattern examined visually. In addition, we used hot spot analysis on these regions allowing hot spot groupings of more preferred values/relationships and cold spots indicating groupings of less preferred values/relationships. Then local autocorrelation was done on this data to identify clusters and outliers of high priority and low priority postcodes. 


\subsection{Diabetes-related PHC and PPH}

Diabetes-related primary health care (PHC) service utilisation in 2013/14 is measured by the number of patients in each postcode who have made one or more Medicare claims for diabetes-related care, based on methods recommended by the Australian Institute of Health and Welfare (AIHW 2009). Specifically, patients receiving diabetes-related care were selected using MBS items for 'General practitioner consultation involving completion of minimum requirements of an annual cycle of care for a patient with diabetes', and at least two pathology tests for $\mathrm{HbAlc}$ and/or fructosamine. Additionally, patients receiving diabetes-related care were also selected using PBS items related to insulin, oral hypoglycaemic agents or blood glucose indicator strips (AIHW 2009). Both MBS and PBS data were combined to identify prevalent patients.

Diabetes-related potentially preventable hospitalisations (PPH) between $2013 / 14$ to $2014 / 15$ were identified from the WA Hospital Morbidity Data System (HMDS) based on either their principal diagnosis being identified by the National Healthcare Agreement (AIHW 2014) as a diabetes-related PPH or identification by Davis et al. (2005) as associated with increased risk for people with diabetes.

From these PHC and PPH prevalent cases for each postcode were obtained. Because at least 5 patient counts were required for the SRR calculations, postcodes that experienced less than 5 counts of health service use were identified as nodata and were excluded from the analysis and visualisations. This can potentially contribute to some bias in the geospatial analysis, particularly when neighbourhoods comprising nodata are used to identify clusters and patterns. Hence, some of the mapped results show nodata postcode areas where the statistical significance of results may be too low.

\subsection{Geospatial analysis and geographic patterns}

The methods used to identify geographic patterns were hotspot analysis and Anselin Local Moran's I autocorrelation analysis.

The hotspot analysis involved the use of the Getis-Ord Gi* statistic which gives a global overview of where there are statistically significant spatial clusters of high values (hot spots) and low values (cold spots) (Getis and Ord 1992, Ord and Getis 1995, Duckett and Griffiths 2016). In essence, this identifies where there are clusters of SRRs that are significantly lower than, similar to or significantly higher than the benchmark region average.

The Local Moran's I statistic (Anselin 1995) of spatial association identifies association of, in this case, SRR values of postcodes, with the values of neighbouring postcodes. It can identify statistically significant clusters of high values $(\mathrm{HH})$ and low values (LL) as well as identify outliers of high values surrounded by low values (HL) and low values surrounded by high values (LH). These results provide a more local perspective on clusters than the hotspot analysis which is more global.

\subsection{Comparison with benchmark regions}

The SRRs provide a comparison of health service utilisation rate in a postcode to the average of that for a benchmark region. Comparison to the State average is a well-accepted practice (Duckett and Griffiths 2016).
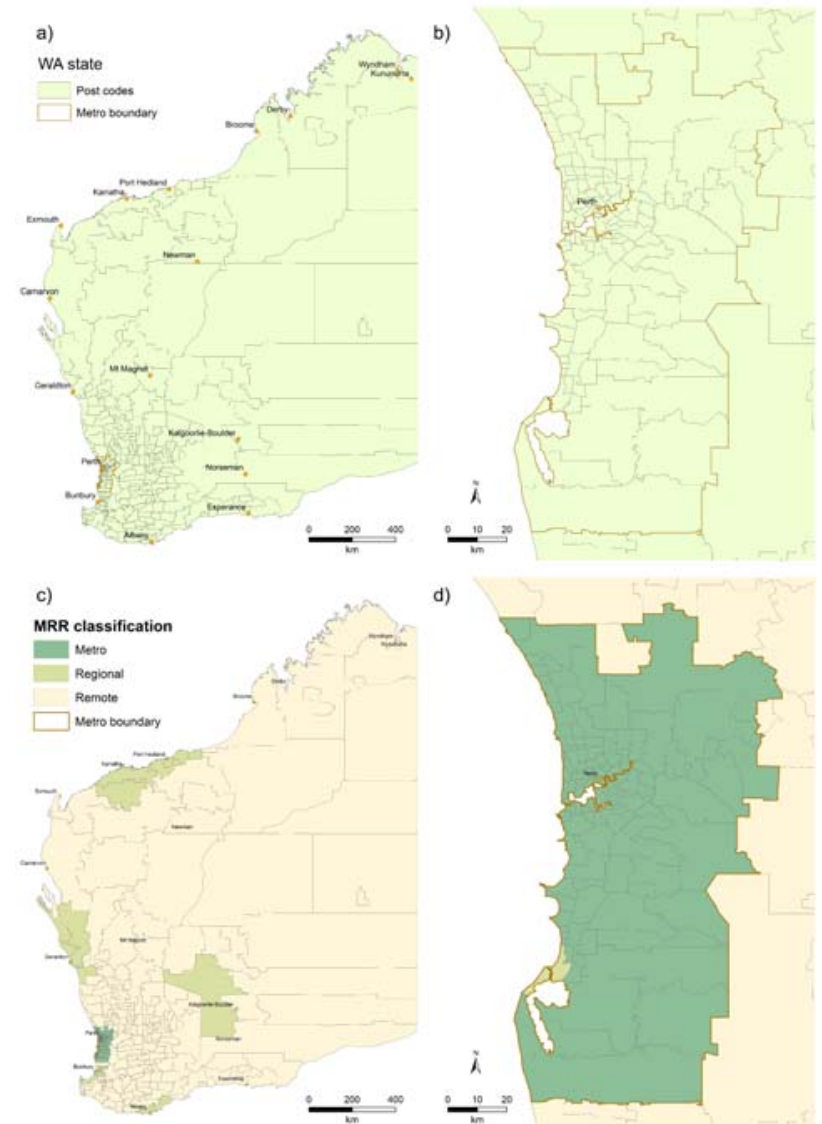

d)

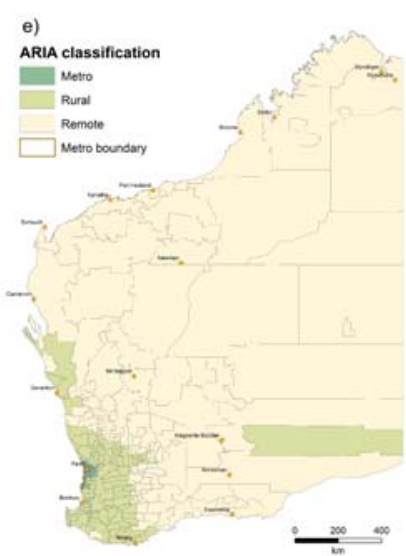

f)

(x)
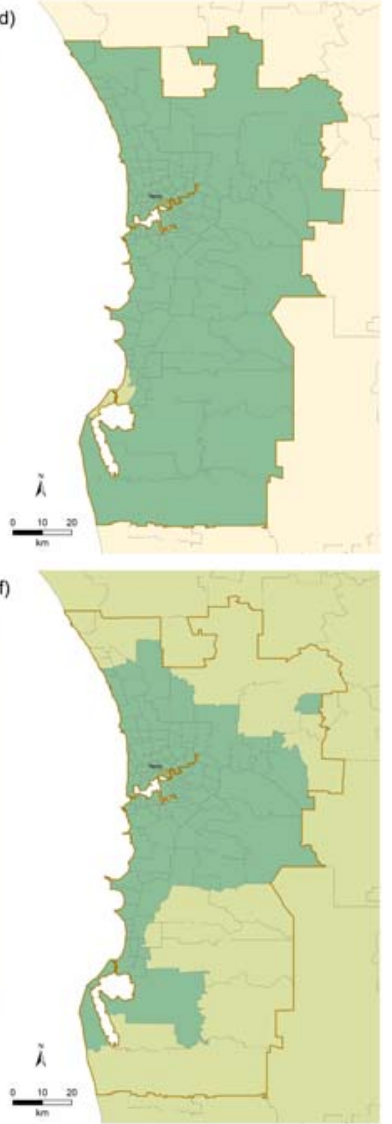

g)

h)

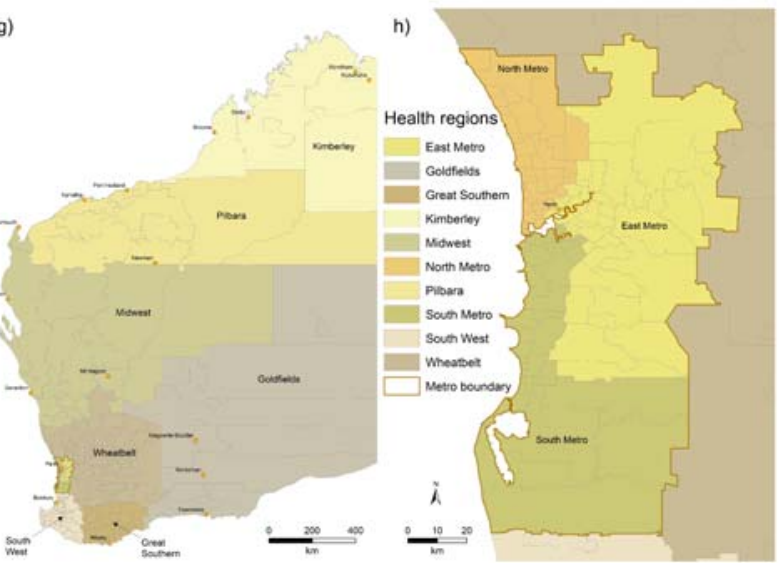

Figure 2. Geospatial boundaries of benchmark regions: WA state $(\mathrm{a}, \mathrm{b})$, Metro/regional/rural regions (c,d), ARIA-based regions (e,f) and Health Regions ( $g, h)$ 
Biases due to differences in population age structures are removed by using age standardised rates. However, there are other factors that can influence the use of health services, including lifestyle risk factors, affordability, remoteness from services, etc. (Falster and Jorm 2017). Some of the geographic variation in potentially preventable hospitalisation may reflect these differences in the population.

Because of differences in the underlying risk factors and disease prevalence in the specific type of region chosen, the results of SRRs can therefore be sensitive to those regions. To determine the sensitivity to different groupings of regions (as detailed in Figure 1), we decided to calculate SRRs for each postcode compared to its regional rate and to compare their spatial variations and patterns.

The regions we used as benchmarks are shown in Figure 2 which shows maps at two scales, one for the state and another for the Perth metropolitan area. The region benchmarks are:

1. State - the state of Western Australia (WA). This would compare each postcode to the WA state average.

2. Metropolitan/Regional/Rural (MRR) - the state subdivided into metropolitan (Perth), regional and rural areas. Five regional centres were chosen as they had reasonable provision for health services and relatively large populations (eg. of more than 30,000).

3. ARIA-based regions - the state subdivided into three regions - metropolitan, rural, remote - based on the ARIA+ accessibility classifications. Specifically, 'metropolitan' region includes 'highly accessible' ARIA; 'regional' region includes 'accessible' and 'moderately accessible' ARIA; 'remote' region includes 'remote' and 'very remote' ARIA.

4. Health Regions - based on the Department of Health Western Australia administrative health regions.

As previously mentioned (refer Section 1.2), Gavidia et al. (2018) identified differences in PHC service utilisation and PPH between the metropolitan and rural areas of the state. Hence, by comparing postcode rates to the State average, there would be an expectation that these two areas would exhibit marked differences in their spatial patterns. Considering also that geographic variations exist among the rural areas, which do vary by remoteness and accessibility (Gavidia et al. 2018), we explored these different region benchmarks.

Given the obvious differences between the metropolitan and rural areas of the state, we examined PPHs relative to geographic regions that distinguish differently between metropolitan and rural areas. Additionally, we used the available feedback from General Practice surveys obtained through the MBS/PBS Project (Gavidia et al. 2018) which suggested that in addition to considering metropolitan versus rural regions, built-up regional centres should also be distinguished. We subsequently identified 5 regional centres in addition to the metro area and the remaining postcodes in rural areas and compared PPHs to their averages (Figures $2 \mathrm{c}$ and $2 \mathrm{~d}$ ).

Remoteness and accessibility to health services does impact medical care and hospitalisations (Breadon 2013, DOHWA 2017). Therefore, we also explored the rural/urban divide using the Accessibility/Remoteness Index of Australia (ARIA) national standard classification that defines 5 categories of remoteness based on physical road distance to the nearest urban centre. We redefined these categories into 3 classes (Figures $2 \mathrm{e}$ and 2f), namely - metropolitan, rural and remote - that roughly equate to the 3 MRR classes, so that we could compare the analysis with the MRR regions.

Finally, we also used the administrative Health regions utilised by the Department of Health Western Australia. These are 10 regions selected on the basis of a measure of homogeneity among the health populations within each region (Figures $2 \mathrm{~g}$ and $2 \mathrm{~h}$ ). Three (3) of the regions are in the metropolitan area and 7 in the regional and remote areas of the state.

\section{RESULTS AND DISCUSSION}

The results of mapping and geospatial analysis of PPH and PHC comparisons among postcodes are outlined in this section. We initially detail the outcomes for PHCs followed by that for $\mathrm{PPHs}$ and then present the outcomes by exploring the relationship between $\mathrm{PHC}$ and $\mathrm{PPH}$.

\subsection{Results and discussion of PHC analysis}

3.1.1 Mapping PHC relative to regions: For 2013/14 financial year (ie, 1 July 2013 to 30 June 2014) there were a total of 110,499 patients (including missing postcodes) in Western Australia who have used diabetes-related primary health care services.

When compared to the state, PHCs are relatively low in some rural areas including the far north and central north (eg. Kimberly and Pilbara) as well as southwest areas (Figures 3a and $3 \mathrm{~b}$ ). There are some areas that experience high PHC across the central rural areas. The central and east metropolitan areas tend to have low PHCs with some high PHCs in the middle suburbs.

When compared to the MRR regions benchmark (Figures $3 \mathrm{c}$ and 3d), the PHCs become higher in some areas to the south of the state, and around the already high PHCs in the metropolitan areas, but remain mostly unchanged for the rest of the state and metropolitan areas. Overall there is a slight pronouncement of high PHC areas. When compared to the ARIA-based regions benchmark (Figures $3 \mathrm{e}$ and $3 \mathrm{f}$ ), there were some small adjustments in the outer metropolitan and mid rural areas but little elsewhere, relative to the MRR benchmark results.

This suggests that the ARIA-based regions are a better representation than the MRR regions, when used as a benchmark, and hence are a better basis for comparing PHCs. Therefore, it was decided not to include comparisons with the MRR regions in subsequent analysis of PHCs.

When using the HRs as a benchmark for comparison, the PHCs in the central and Pilbara regions changed from higher to more similar rates (Figures $3 \mathrm{~g}$ and $3 \mathrm{~h}$ ). Rather than seeing more concentrated clusters of postcode areas with high PHC, there are more differences among local postcode areas to distinguish them within their more localised (HR) regions.

3.1.2 PHC hotspot analysis: We conducted hotspot analysis on the PHC results to visualise the distribution of high and low PHC usage. The hotspots were generated from the clusters of 1's, 2's and 3's representing the SRRs of PHCs significantly lower than, similar to or significantly higher than the benchmark averages. Similarly, comparisons were made with different benchmarks of State, ARIA-based regions and HRs. 


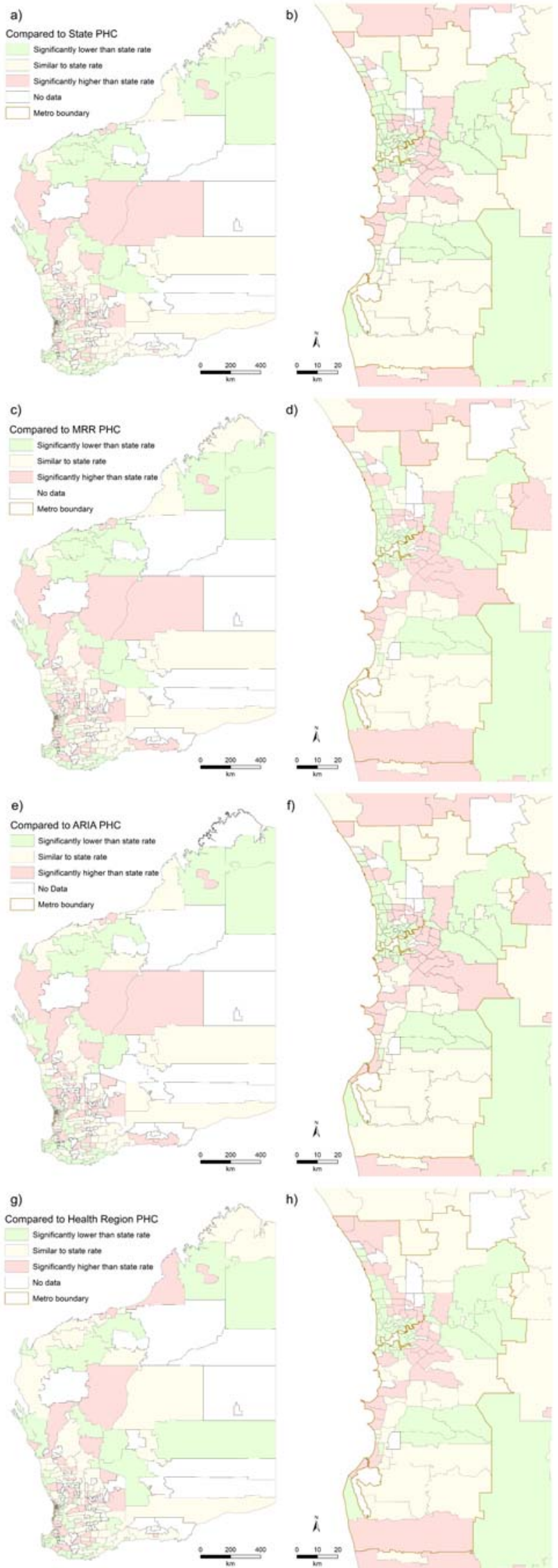

Figure 3. PHC at state/metro scales for regions: State (a,b), MRR (c,d), ARIA-based (e,f) and Health Region (g,h)
The results are shown in Figure 4. For PHCs relative to the state (Figure 4a) there is a band of hotspots in the central regional area indicating higher PHCs relative to the state. There are low PHCs found in two bands along the northwest and southwest coasts. When the ARIA-based regions were used as the benchmark, (Figure 4b), the hotspot band was slightly thinned out and the northwest cold spot disappeared.

This trend continues with using HR as the benchmark (Figure 4c) where the hot spot is further refined. The indication is that the PHC hot and cold spots tend to dwindle as they are compared with smaller and more local region averages. Yet there is a core central rural region that does have relatively higher PHC; this is consistent with the mapping of $\mathrm{PHC}$ in Figure 3.
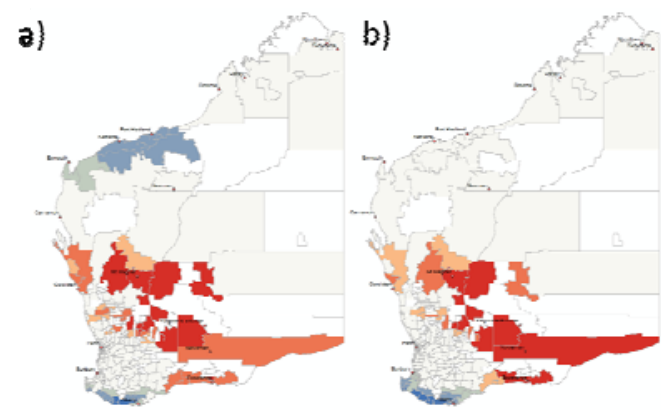

c)

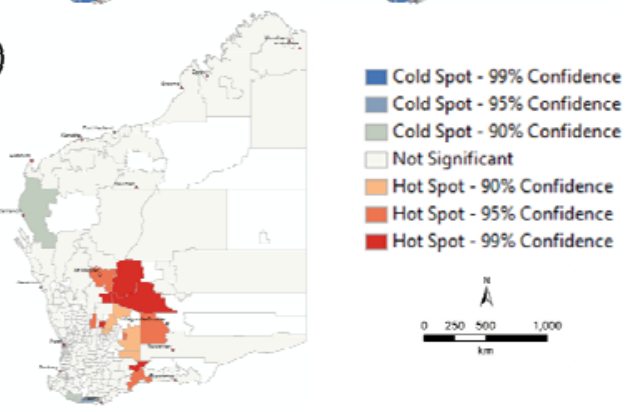

Figure 4. PHC hotspot analysis relative to a) State, b) ARIAbased regions and c) Health Regions

3.1.3 PHC local autocorrelation analysis: The local autocorrelation analysis for PHC relative to the State average (Figures $5 \mathrm{a}$ and $5 \mathrm{~b}$ ), which shows the results relative to the local neighbouring postcodes, identified a large cluster of postcode areas with high PHCs through a band from northwest to southeast of postcodes in south central WA. There are clusters of postcode areas with low PHCs in the Pilbara, southwest along the coast and Perth central and northern areas. There are some high outliers postcode areas in the outer metropolitan areas as well as scattered along the rural north coast and southwest.

For the ARIA-based regions (Figures $5 \mathrm{c}$ and $5 \mathrm{~d}$ ), the analysis results changed the Pilbara to be non-significant and highlighted some further outliers along the Midwest coast and southwest. The metropolitan area showed little change. Some high PHC outliers were found north of Perth and in the southeast, with some low outliers in the southwest interior. The clusters of postcode areas are similar to those identified when using the state as a benchmark.

The HR analysis (Figures 5e and $5 \mathrm{f}$ ) shows a different pattern with high clusters of PHC in the Midwest and low clusters in the metropolitan central areas. Then there is a large area of low 
PHC outliers in the central rural area and high PHC outliers in the central northwest in relation to two of the HRs.

Although the classifications may vary, it is many of the same postcodes that are being highlighted in clusters and outliers. This may be a reason to further investigate the PHC needs of these postcode areas. There may be underlying population and health care factors within these postcode areas with the outcome that they are persistantly highlighted in the various hotspot analyses.

a)
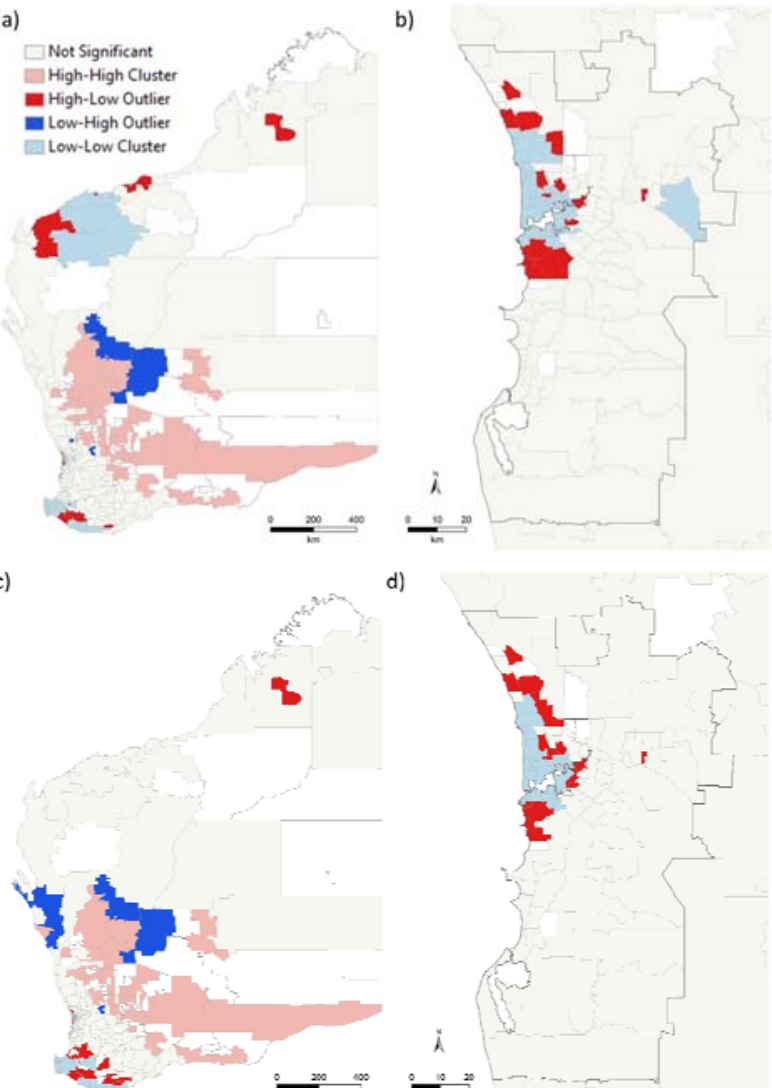

e)

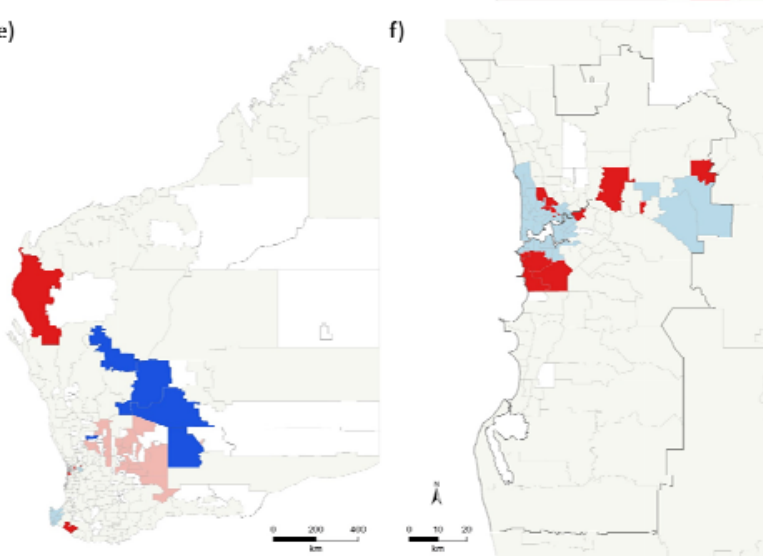

Figure 5. PHC local autocorrelation analysis relative to State (a,b), ARIA-based regions (c,d) and Health Regions (e,f)

\subsection{Results and discussion of PPH analysis}

3.2.1 Mapping PPH relative to specific type of region chosen: For the period 2013/14 to 2014/15 financial years, there were a total of 24,712 (including missing) PPHs for Western Australia. The postcode areas were mapped based on the SRRs of each postcode compared to its state or regional average, based on each of the four types of regions selected (see Section 2.4) (Figure 6).

Figures $6 \mathrm{a}$ and $6 \mathrm{~b}$ show a large number of remote areas with PPHs higher than the state average whereas most regional areas between Geraldton and Albany have a PPH rate similar to the state average. The postcodes with PPH lower than the state average are mostly in the central metropolitan area and around some of the main regional centres including Bunbury, Albany and Geraldton.

When postcode areas were mapped based on the SRRs of each postcode compared to its corresponding MRR regions as shown in Figures $6 \mathrm{c}$ and 6d, many of the central rural postcodes change from higher/similar to similar/lower. Conversely, some of the postcodes in the outer metropolitan areas changed from similar to higher than the regional average.

When using the ARIA-based regions (Figures 6e and 6f) the metropolitan region showed some higher rates in coastal postcode areas. Some of the central rural postcode areas changed from higher to lower/similar PPH rates. Interestingly, use of the ARIA-based regions had a greater effect on the central rural regions, with little effect on the metropolitan and remote rural regions, relative to the use of MRR. This is most likely due to the fact that the metropolitan-rural-remote divide is based on true (road distance) accessibility as per the ARIA definition

This suggests that the ARIA-based regions are a better representation than MRR regions when used as a benchmark for comparing PPHs. Therefore, it was decided not to include comparisons with the MRR regions in subsequent analysis of PPHs, similar to our decision for PHCs (Section 3.1.1).

Finally, we showed PPHs for the postcode areas relative to the administrative Health Regions used by the Department of Health Western Australia (Figures $6 \mathrm{~g}$ and $6 \mathrm{~h}$ ). For the metropolitan area, the postcodes to the north and east of the city centre had fewer higher PPH rates compared to its corresponding ARIA-based rate and the central and southern postcodes remained largely unchanged. In the remote areas, there was a change from higher to more similar PPHs. There were also some changes in central rural areas from lower to similar PPH rates. With the comparison to the local regions, the HRs in this case, the postcode areas appear not to be getting masked out as much as when their PPHs are benchmarked with the broader ARIA-based regions. 


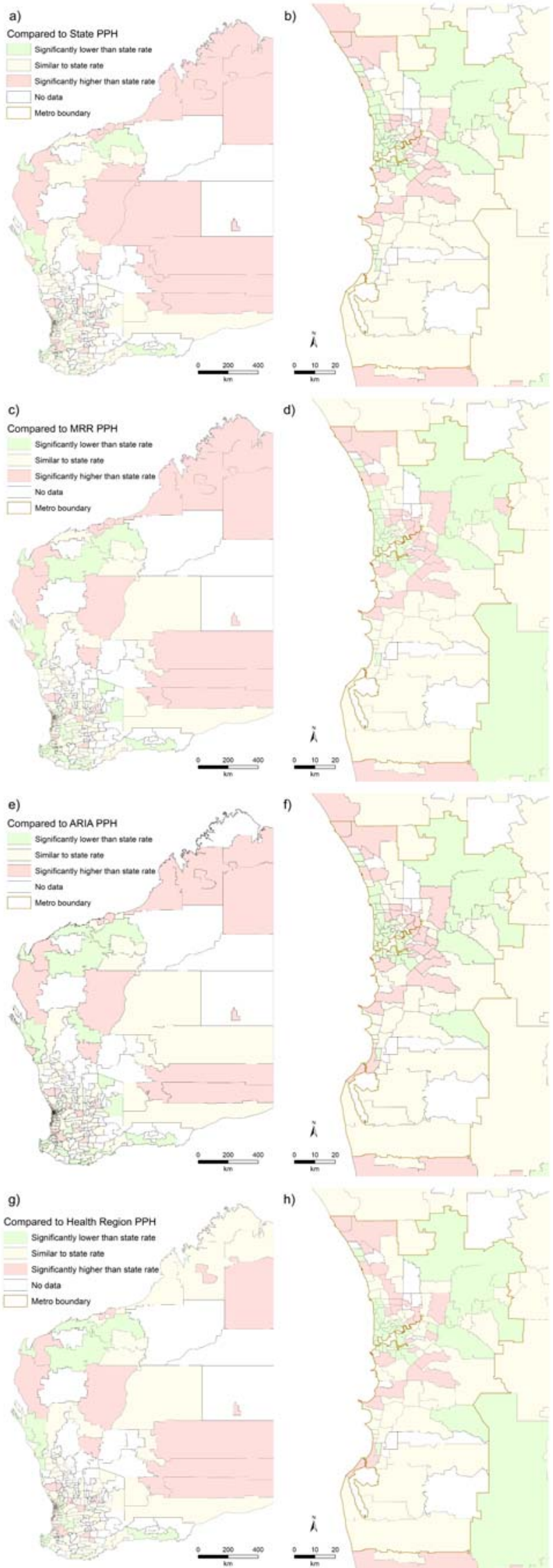

Figure 6. PPH at state and metro scales relative to State $(\mathrm{a}, \mathrm{b})$, MRR (c,d), ARIA-based regions (e,f) and Health Regions (g,h)
3.2.2 PPH hotspot analysis: Hotspot analysis was performed in order to visualise global trends in the geographic distribution of PPHs across the state. This was conducted for the values relative to the State, ARIA-based regions and Health Regions. As already mentioned, the MRR regions were removed from the analysis.

The hotspot analysis results are shown in Figure 7. The red areas indicate high values (hot spots) of PPHs and the blue areas indicate low values (cold spots) of PPHs.
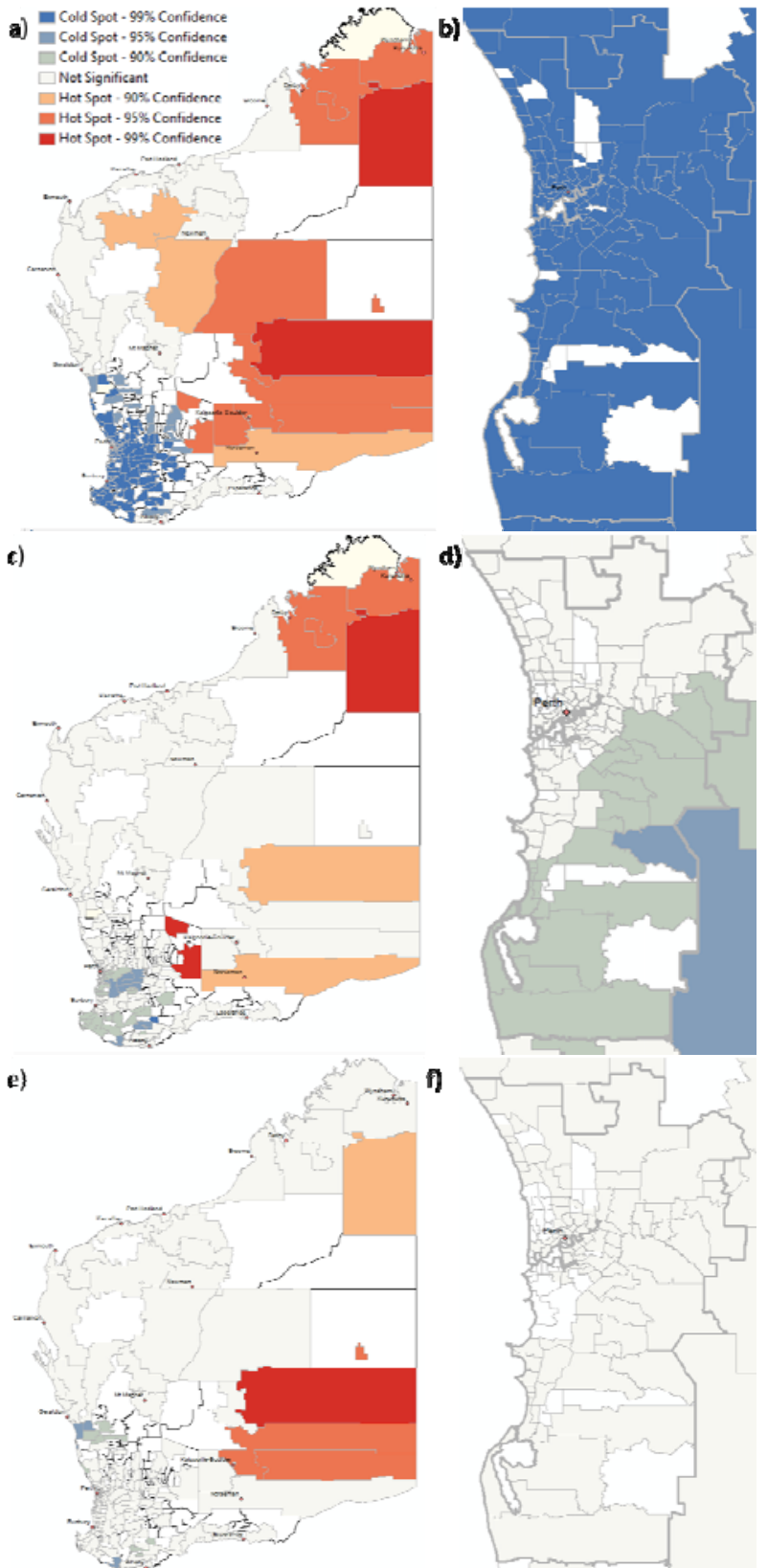

Figure 7. PPH hotspot analysis relative to State $(a, b)$, ARIAbased regions (c,d) and Health Regions $(\mathrm{e}, \mathrm{f})$

Notice how, in Figures $7 \mathrm{a}$ and $7 \mathrm{~b}$, the metropolitan area has distinctly lower PPHs than the state average whereas the rural regions, particularly those that are more remote (eg. Eastern side of WA) have PPHs higher than the state average. This corroborates the visual inspection of Figures $6 \mathrm{a}$ and $6 \mathrm{~b}$. 
When observing the hot spots using ARIA-based regions (Figures $7 \mathrm{c}$ and $7 \mathrm{~d}$ ), the central rural areas begin to show up as non-significant with some slightly higher and lower hotspots of $\mathrm{PPH}$. The central and northern metropolitan areas likewise appear as non-significant.

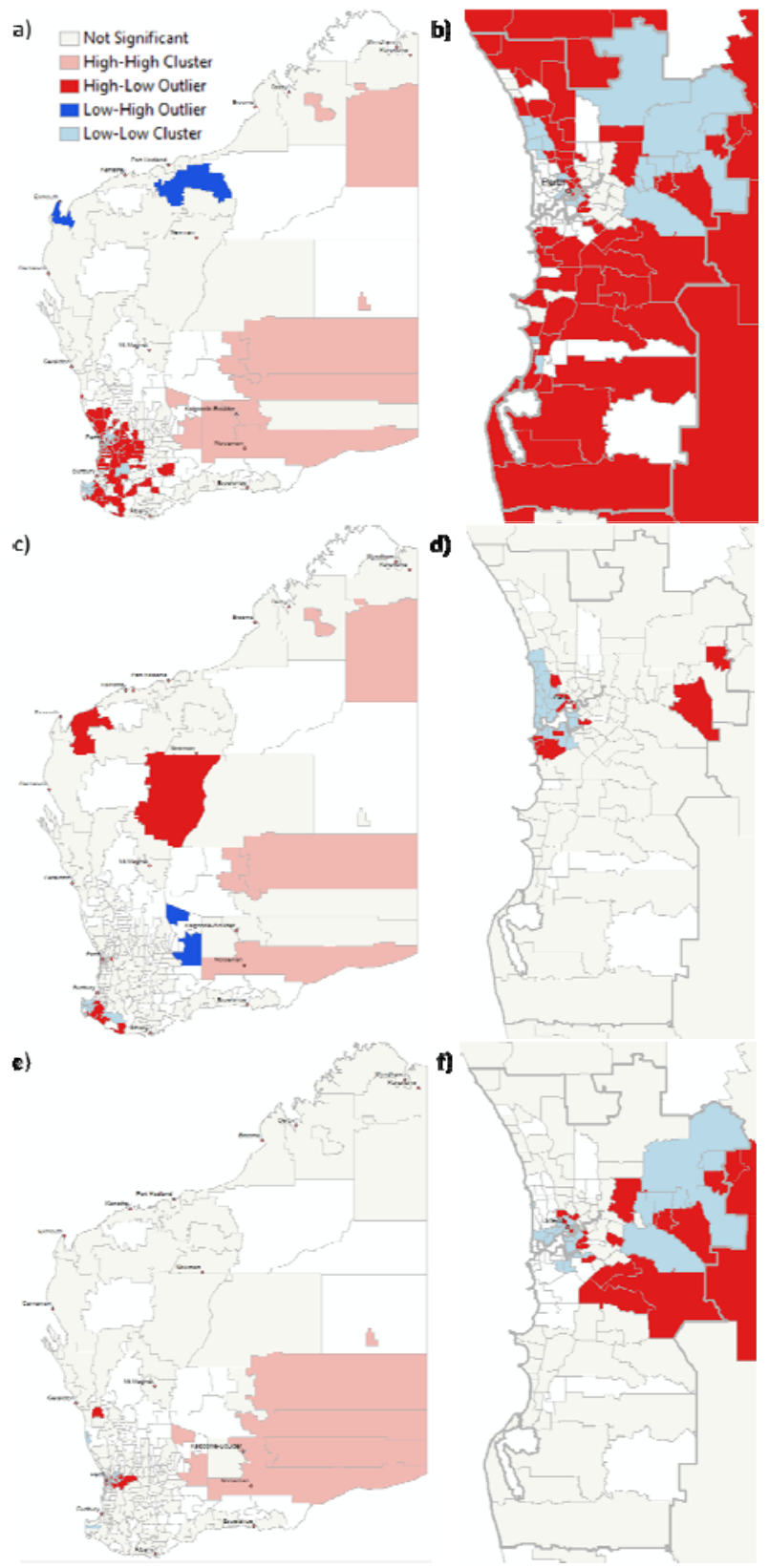

Figure 8. PPH local autocorrelation analysis relative to a) State $(\mathrm{a}, \mathrm{b})$, ARIA-based regions (c,d) and Health Regions (e,f)

In Figures $7 \mathrm{e}$ and $7 \mathrm{f}$ where the PPHs are compared to the more local HRs, most of the state is non-significant with some hotspots in the remote rural areas and some cold spots in the western central areas. Note how the far northeast (Kimberley) region does not stand out as a hot spot and nor does the metropolitan region stand out as a cold spot. The postcode areas are clearly identified as higher or lower where PPH stands out relative to the more localised health region benchmark.
3.2.3 PPH local autocorrelation analysis: The local autocorrelation analysis for PPH for the State (Figures $8 \mathrm{a}$ and $8 b$ ), which shows the results relative to the local neighbouring postcodes, identified a number of high outliers (HL) throughout the southwest and Perth metropolitan regions. Low outliers (LH) were found in the north central regions (Pilbara). Low clusters (LL) were found in the northeast metropolitan areas and high clusters $(\mathrm{HH})$ in the remote eastern regions of the state.

For the ARIA-based region benchmark, analysis showed less of a contrast between metropolitan and rural areas (Figures 8c and 8d) but still showing high clusters in remote regions and low clusters in some southwest regions and west central metropolitan region. Some outliers are shown in the central rural areas and some central metropolitan postcode areas.

For the autocorrelation analysis using HR as a benchmark, most of the state and metro areas were shown as not significant (Figures 8e and 8f). High clusters of PPH are found in the southeast remote areas and low clusters in the northeast metropolitan areas, consistent with analysis using the ARIAbased regions as benchmark. A few high outliers were found in postcode areas relatively close to the metropolitan area. These results show that PPH is similar among neighbouring postcode areas apart from some clusters and a few outliers, as mentioned.

\subsection{Analysing relationships between PHC and PPH}

The relationship between PHC and PPH was first explored by mapping the five possible relationships (identified in Section 2.1 ) and visually examining the geographic pattern. Figure 9 shows the resulting maps for comparisons with each of the 3 benchmarks.
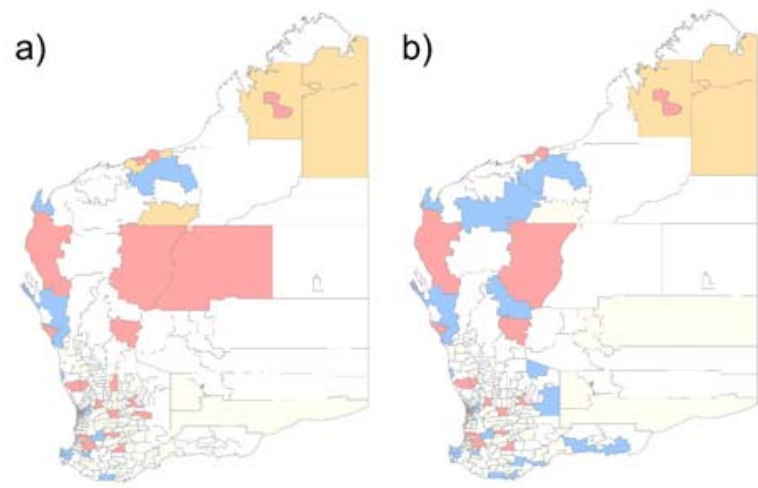

c)
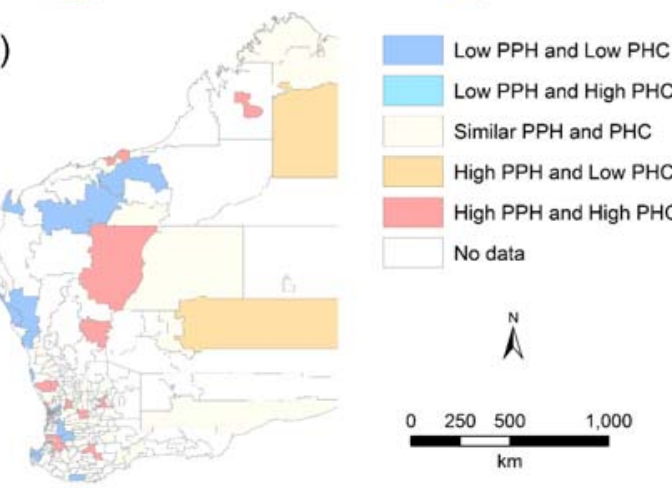

Figure 9. Relationship of PHC to PPH relative to State (a,b), ARIA-based regions (c,d) and Health Regions (e,f) 
a)

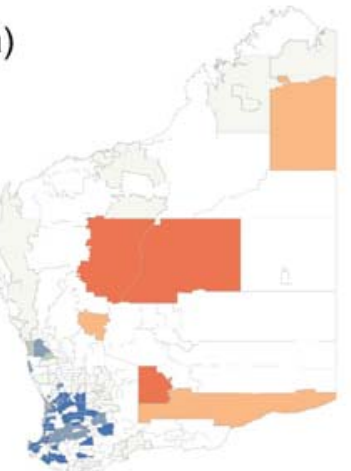

c)

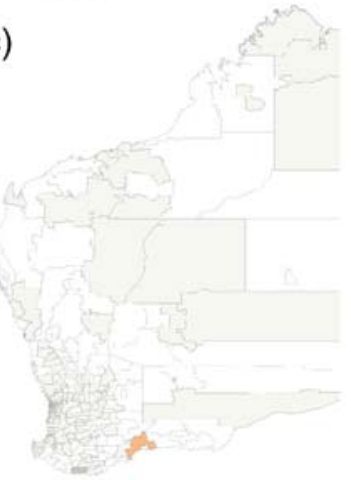

b)

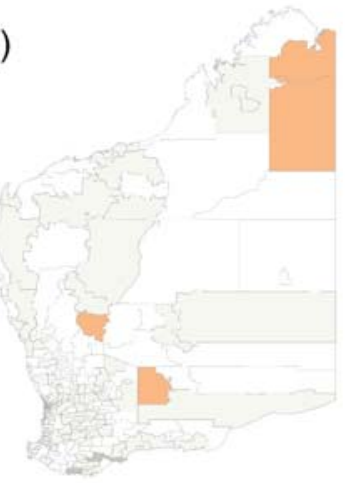

Cold Spot - $99 \%$ Confidence Cold Spot - $95 \%$ Confidence Cold Spot - $90 \%$ Confidence Not Significant

Hot Spot - $90 \%$ Confidence Hot Spot - 95\% Confidence Hot Spot - $99 \%$ Confidence

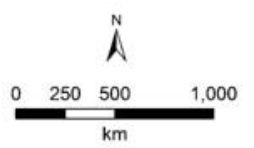

Figure 10. Hotspot analysis of relationship of PHC to PPH for State (a), ARIA-based regions (b) and Health Regions (c)

Some of the high PHC and high PPHs are found in the central Midwest areas of WA and scattered among postcodes within a few hundred kilometres of the metropolitan area. Some of the low PHC and low PPH postcode areas are found in the Pilbara and central west coast. These relationships are generally persistent in the analysis using the three different benchmarks. This then gives an indication that these postcode areas may be explored further to better understand their population and health service's needs.

The hotspot analysis of the relationship of PHC to PPH showed in a global sense (Figures 10a and 10b) that there were hot spots of less preferred relationships of PHC and PPH in the rural regions and cold spots of more preferred relationships in the southwest area (refer to Section 2.1 on explanation of 'preferred'). Although not shown in the maps, the metropolitan areas also showed cold spots when benchmarked with the state average indicating the more preferred relationships of PHC to PPH. Interestingly, this information faded away when the ARIA-based regions and more local HR benchmark was used (Figures 10c and 10d).

The local autocorrelation results for the state level (Figures 11a and 11b) show clusters of less preferred $\mathrm{PHC} / \mathrm{PPH}$ relationships in some of the rural areas. The outer metropolitan region shows outlier postcodes of less preferred $\mathrm{PHC} / \mathrm{PPH}$ relationships. Interestingly, as the local regions are used in the analysis, this information fades away for both rural and metro postcodes in the analysis of more local regions (ARIA-based regions and HR) (Figures 11c and 11d). This suggests that benchmarking with regions that are too localised may even miss some information about the relationship of PHC to $\mathrm{PPH}$.

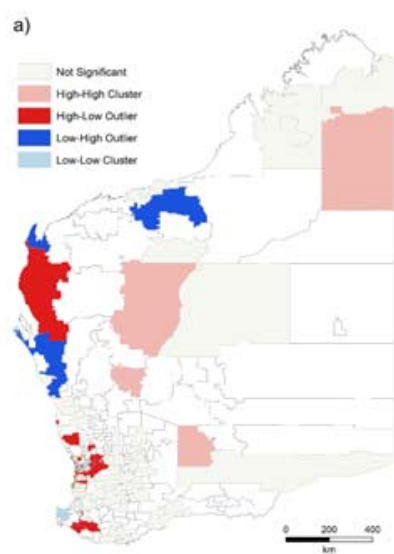

b)

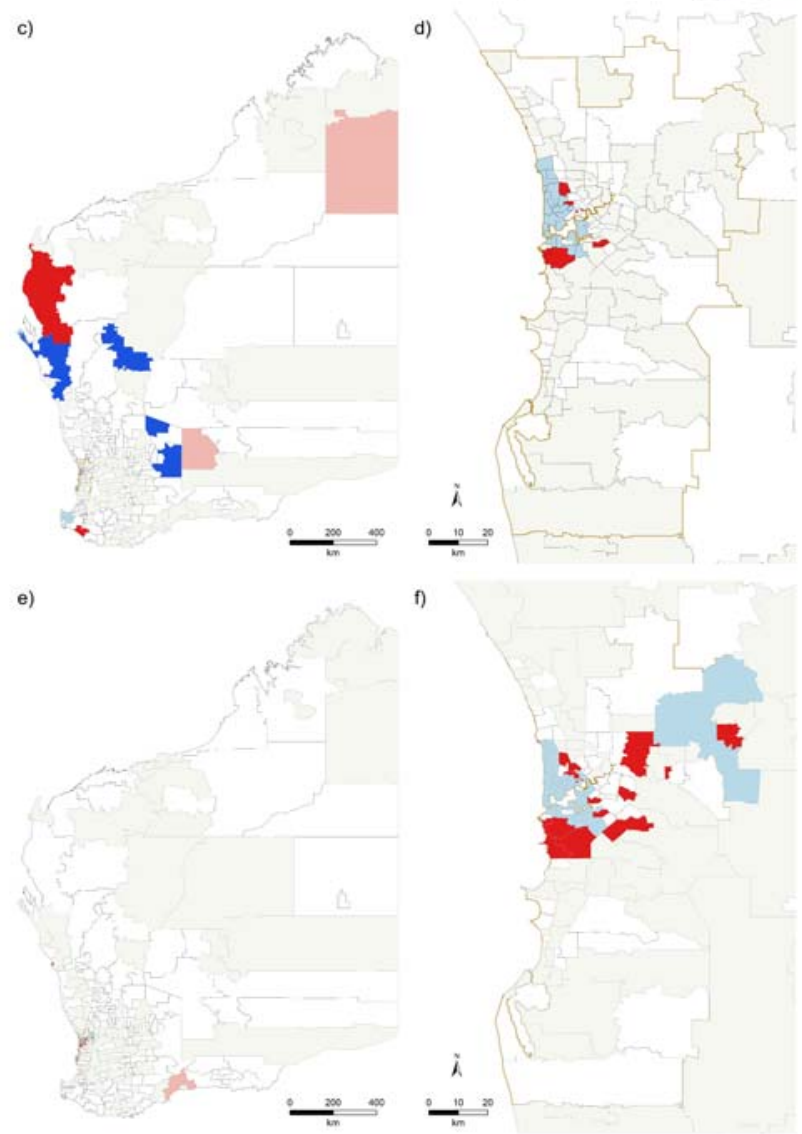

Figure 11. Local autocorrelation analysis of relationship of $\mathrm{PHC}$ to PPH

\section{CONCLUSIONS}

When exploring the geographic variation among PHC and PPH by mapping the significant SRR values relative to different benchmarks, the selected benchmark is important and results in potentially different outcomes. Comparing to larger regions provides more of a global view but masks out more of the local differences. Comparing to local regions identifies postcode areas in the context of their more local geographic vicinity (more localised regions). Given that the ARIA-based regions better represented the accessibility to medical services than MRR regions based on five regional centres, it was decided to discontinue the analysis with the latter. 
The global/local differences were evident, for example, for the State region (global) in comparison to using Health Regions (local). To illustrate, there were 59 postcodes for the State region versus 48 postcodes for the HR exhibiting significantly higher PPH and their geographic distribution was quite varied across WA although much less so at the Perth metropolitan level (Figures $3 \mathrm{a}$ and $3 \mathrm{~g}$ ). As a further example, the number of postcodes where PPHs were significantly lower was similar (eg. 61 and 62, respectively) for State and HR, but the geographic variation differed significantly in the rural areas (Figures 3a and $3 \mathrm{~g})$.

The use of different spatial units to benchmark the PHC and PPH values of postcodes assists in evaluating whether the variations in these postcode values are real or attributable to the spatial regions used. Smaller spatial regions may draw out patterns and relationships that are masked out by larger regions, as was the case with both $\mathrm{PHC}$ and $\mathrm{PPH}$ variations. Persistence among multiple spatial units may indicate postcodes that have PHC needs that can help to improve PPHs. The outcomes of geographic variation using different spatial units can identify those postcodes to be considered for further exploration.

The hotspot analysis provides a more general overview of the underlying relative pattern of low and high PPHs and PHC utilisation. These relative patterns may vary when different benchmark spatial regions are used, but the persistence of postcodes among the benchmark regions may provide an indication that those postcodes may be of interest for further exploration.

The local autocorrelation results indicated if a postcode is part of a cluster (eg. similar to neighbouring postcode areas) or is an outlier (ie. different from neighbouring postcode areas). These postcodes can be considered for further exploration of PHC needs and reduction of PPHs. The analysis relating PHC to PPH identified more and less preferred relationships, but some of these associations appeared not to show up with localised benchmark regions. Also, the consideration of what relationships are preferred needs more examination and research. Further work needs to be undertaken as to how best to draw out the relationships of PHC to PPH.

\section{ACKNOWLEDGEMENTS}

This work has been supported by the Cooperative Research Centre for Spatial Information, whose activities are funded by the Business Cooperative Research Centres Programme. The authors acknowledge the support and facilities provided by Curtin University and the Department of Health Western Australia. We also acknowledge the Commonwealth Department of Health for providing the Western Australian MBS/PBS data for the project.

\section{REFERENCES}

AGDOH, 2011. Medicare Benefits Schedule. Australian Government Department of Health, http://www.health .gov.au/internet/hta/publishing.nsf/content/mbs-1 (13 July 2018).

AGDOH, 2018. About the PBS. Australian Government Department of Health, http://www.pbs.gov.au/info/about-thepbs (13 July 2018).
AIHW, 2009. Diabetes prevalence in Australia: an assessment of national data sources. Australian Institute of Health and Welfare, Diabetes series no. 12. Cat. no. CVD 46, Canberra.

AIHW, 2014. National Healthcare Agreement: PI 18-Selected potentially preventable hospitalisations. Australian Institute of Health and Welfare,

http://meteor.aihw.gov.au/content/index.phtml/itemId/517648 (22 June 2018).

Anselin, Luc, 1995. Local Indicators of Spatial AssociationLISA. Geographical Analysis 27(2): 93-115.

Breadon, P., 2013. Access all areas: new solutions for GP shortages in rural Australia. Grattan Institute.

Davis WA, Knuiman MW, Hendrie D, Davis TM, 2005. Determinants of diabetes-attributable non-blood glucoselowering medication costs in type 2 diabetes: the Fremantle Diabetes Study. Diabetes Care, 28:329-336.

DOHAC, 2001. Measuring Remoteness: Accessibility/ Remoteness Index of Australia (ARIA). Department of Health and Aged Care, Revised Edition, Occasional Papers: New Series Number 14, October,

http://www.health.gov.au/internet/main/publishing.nsf/Content/ E2EE19FE831F26BFCA257BF0001F3DFA/\$File/ocpanew14. pdf (12 July 2018).

DOHWA, 2017. Lessons of Location: Potentially preventable hospitalisation hotspots in Western Australia 2017. Government of Western Australia Department of Health.

Duckett, S., \& Griffiths, K., 2016. Perils of place: identifying hotspots of health inequalities. Grattan Institute.

Falster, M. \& Jorm, L., 2017. A guide to the potentially preventable hospitalisations indicator in Australia. Centre for Big Data Research in Health, University of New South Wales in consultation with Australian Commission on Safety and Quality in Health Care and Australian Institute of Health and Welfare: Sydney; March 2017.

Gavidia, T., Varhol, R., Xiao, A., Koh, C., Mai, Q., Liu, Y., Turdukulov, U., Parsons, S., Fievez, P., Veenendaal, B., Somerford, P. 2018. Geographic variation in primary health care service utilisation and potentially preventable hospitalisations in Western Australia. Department of Health WA, Cooperative Research Centre for Spatial Information, Curtin University and WA Primary Health Alliance. Perth: Western Australia, in press.

Getis, A., Ord, J.K., 1992. The Analysis of Spatial Association by Use of Distance Statistics. Geographical Analysis, 24

Ord, J.K., Getis, A., 1995. Local Spatial Autocorrelation Statistics: Distributional Issues and an Application. Geographical Analysis, 27.

Rothman, K.J. \& Greenland, S. 1998. Modern Epidemiology. $2^{\text {nd }}$ Edition, Lippincott-Raven Publishers. 